\title{
Effect of Fermented Traditional Chinese Prescription With Lactobacillus Plantarum on Dysbacteriotic Diarrhea Mice Induced by Ceftriaxone Sodium
}

\author{
Xin Guo \\ Shanxi Agricultural University \\ Zipeng Yan \\ Shanxi Agricultural University \\ Jixiang Wang \\ Shanxi Agricultural University \\ Xinfeng Fan \\ Shanxi Agricultural University \\ Jie Kang
}

Fenyang College Shanxi Medical University

Ruiyan Niu

Shanxi Agricultural University

Zilong Sun ( $\square$ sunzilong2000@outlook.com )

Shanxi Agricultural University https://orcid.org/0000-0003-4907-7271

\section{Research Article}

Keywords: Antibiotic, TCM, FTCM, Intestinal flora and barrier

Posted Date: November 12th, 2021

DOI: https://doi.org/10.21203/rs.3.rs-1052330/v1

License: (a) (i) This work is licensed under a Creative Commons Attribution 4.0 International License. Read Full License 


\section{Abstract}

Background: Buzhongyiqi decoction (B), Sijunzi decoction (S), and Shenlingbaizhu decoction (SH) have been extensively clinically used for the treatment of health status and diseases caused by spleen-qi deficiency for many years and microbial fermentation has been widely applied in Traditional Chinese Medicine (TCM) for thousands of years in China. This study was aimed to investigate the mitigative effect of TCM and fermented TCM (FTCM) with Lactobacillus plantarum (LP) in antibiotic-associated diarrhea (AAD), and identify the compounds of $S$ and Fermented $S$ (FS).

Methods: The dysbacteriotic diarrhea mice induced by ceftriaxone sodium (CS) were treated with LP, B, S, $\mathrm{SH}$, Fermented B, S, and SH. The diarrhea indexes, the abundance of some gut bacteria, intestinal morphometrics, and the mRNA expressions related to intestinal barrier function were assessed at multilevels. In addition, S and FS were chosen to identify and relatively quantify the compounds by ultrahigh-performance liquid chromatography coupled with quadrupole time-of-flight mass spectrometry (UHPLC-Q-TOF/MS), and different expressed compounds were analysed.

Results: Results showed that CS significantly increased the fecal output weight, the total number of fecal output, and fecal water content, indicating the occurrence of diarrhea, while TCM, LP, and FTCM alleviated the diarrhea to different degrees and FTCM showed more sustained effects. Then, bacterial culture test showed above symptoms were accompanied with the disruption of some intestinal flora. Meanwhile, the diarrhea mice showed abnormal intestinal morphology and destroyed intestinal barrier manifested as reduced mRNA expression of Aquaporins (AQPs) and tight junction (TJ) protein. Notably, the above indices were alleviated in other treatments mice.

Conclusions: All these findings imply that the intestinal side effects caused by antibiotics can be alleviated by TCM, LP, and fermented TCM through regulating the intestinal flora and barrier function, which provides an idea of further development and application of them in the clinical use of antibiotics.

\section{Introduction}

Antibiotics have been used for the prevention and treatment of lots of severely bacterial infections and saved countless lives. In recent years, a large number of studies have revealed that the abuse of antibiotics may cause undesirable consequences, such as antibiotic resistance, pathogen overgrowth, alteration of gut microbial composition, increased bacterial susceptibility, and the risk of repeated infections[1]. In addition, nephrotoxicity and neurotoxicity[2], emesis, antibiotic-associated diarrhea (AAD) and allergy[3] were also side-effects induced by antibiotics. Notably, antibiotics can destroy the structure of normal gut microbiota and alter the functions in children, adults and animal models, which is dysbacteriosis[4]. Because the gut microbiota shows an important role in immunity, metabolism and endocrinology, the negative impacts of antibiotics on the microbiota lead to further health complications of obesity, allergies, autoimmunity and other diseases[4,5]. The interventions of scientific method are 
effective for improving antibiotic application, which raises a question of whether healthy microbiomes could regulate or restore by probiotics or Traditional Chinese Medicine (TCM).

Some studies have provided important information that probiotics alter gut microbiota and produce flora metabolites that influence health via 1 of 3 general mechanisms: direct antimicrobial effects, enhancement of mucosal barrier integrity, and immune modulation[6]. In addition, TCM also adjusts the balance of intestinal flora[7]. TCM is one of the oldest medicine practices in human history, widely applied to the clinical diagnosis and treatments[8]. In TCM practice, tonic herbs and a few animal sourced medicines are used to strengthen the body and cure diseases caused by Qi deficiency. Some typical Qiinvigorating herbs include Astragali Radix, Ginseng Radix et Rhizoma (ginseng), yam, Codonopsis, Coix seed, Atractylodis Macrocephalae Rhizoma, Glycyrrhizae Radix et Rhizoma (licorice), and Schisandra Chinensis Fructus, and exemplary Qi-invigorating multi-ingredient decoctions (called Tang in Chinese) include “Sijunzi Tang, Lizhong Tang, Buzhongyiqi Tang, and Shenlingbaizhu Tang”[8]. TCMs are mostly relevant to intestinal flora because they inevitably interact with gut microbiota when administrated orally to treat diseases $[9,10]$. Many researchers have investigated metabolites of TCMs by regulating intestinal flora and realized that indeed these metabolites have important pharmacological activities and play various roles in the disease treatment[7]. Indeed, microorganisms have been employed in fermenting TCM, e.g. probiotic was used to ferment Scutellaria Radix[11], Atractylodis Macrocephalae Rhizoma[12], red ginseng[13], and some Chinese prescription, like Danggui Buxue Tang,[14], Sagunja-tang[15], Ge-GenQin-Lian decoction[16].

Based on above, the three kinds of classic prescriptions (Buzhongyiqi decoction, Sijunzi decoction, Shenlingbaizhu decoction) that were fermented by Lactobacillus plantarum were applied to alleviate diarrhea and regulate the imbalance of intestinal flora induced by antibiotic. However, it has not been reported before. In this study, the diarrhea indexes, the abundance of some gut bacteria, intestinal morphometrics, and the mRNA expressions related to intestinal barrier function were assessed at multilevels. In addition, Sijunzi decoction and Fermented Sijunzi decoction due to their better effects were chosen to identify and relatively quantify the compounds by an untargeted ultra-high-performance liquid chromatography coupled with quadrupole time-of-flight mass spectrometry (UHPLC-Q-TOF/MS). The purpose of this study is to investigate whether the intestinal side effects caused by antibiotics can be alleviated by TCM, probiotics, or fermented TCM.

\section{Materials And Methods}

\section{Medicines and strain}

All medicines formulating Buzhongyiqi decoction (B), Sijunzi decoction (S) and Shenlingbaizhu decoction (SH) were purchased from Beijing Tong Ren Tang Co., Ltd. (Beijing, China). Ceftriaxone sodium was purchased from Beijing solarbio science \& technology Co., Ltd. (Beijing, China), and Lactobacillus plantarum (LP) CICC 21809 was obtained from the China Center of Industrial Culture Collection (CICC, Beijing, China). 
Three compounds of B, S and SH were prepared according to Pharmacopoeia of the People's Republic of China. The original composition of $\mathrm{S}$, the four constituting herbs, Radix Codonopsis $(60 \mathrm{~g})$, Atractylodis Macrocephalae Rhizoma (60g), Poria (60g) and Glycyrrhizae Radix et Rhizoma Praeparata (30g), soaked in distilled water at room temperature for $1 \mathrm{~h}$, were decocted twice in $2100 \mathrm{~mL}$ water for periods of $1 \mathrm{~h}$ each in a glass flask[17], and concentrated to $1 \mathrm{~g} / \mathrm{mL}$ by rotary evaporation apparatus (RE3000-A, Shanghai Yarong biochemical instrument factory, China). The B and SH were decocted and concentrated as the above method. The herb compositions of B, S and SH were shown in Table 1.

Table 1

The herb compositions

\begin{tabular}{|ll|}
\hline Name of TCM & Place of production \\
\hline Radix Astragali & Gansu \\
\hline Codonopsis Radix & Shanxi \\
\hline Atractylodes Macrocephala Koidz. & Zhejiang \\
\hline Glycyrrhizae Radix et Rhizoma Praeparata & XinJiang \\
\hline Angelicae Sinensis Radix & Gansu \\
\hline Citrus Reticulata & Zhejiang \\
\hline Cimicifugae Rhizoma & Neimenggu \\
\hline Radix Bupleuri & Shanxi \\
\hline Poria & Hubei \\
\hline Lablab Semen Album & An'hui \\
\hline Rhizoma Dioscoreae & Henan \\
\hline Lotus Seed & Hubei \\
\hline Coicis Semen & Guizhou \\
\hline Platycodon Grandiforus & An'hui \\
\hline Fructus Amomi & Guangdong \\
\hline
\end{tabular}

The strain was inoculated into sterile liquid MRS medium for about $24 \mathrm{~h}$ at $37^{\circ} \mathrm{C}$, then inoculum LP $(3 \%$, $\mathrm{v} / \mathrm{v}$ ) was inoculated into $100 \mathrm{~mL}$ of $\mathrm{B}, \mathrm{S}$ and $\mathrm{SH}$ decoction, respectively, in which $\mathrm{pH}$ was regulated to 6.5 using food-grade $\mathrm{Na}_{2} \mathrm{CO}_{3}$. The fermented decoctions inoculated with LP was incubated at $37{ }^{\circ} \mathrm{C}$ for $24 \mathrm{~h}$ to reach a concentration of $10^{9}$ colony forming units (CFU) $\mathrm{mL}^{-1}$. Non-fermented TCM decoctions were kept at $4{ }^{\circ} \mathrm{C}$ condition and heated to $37^{\circ} \mathrm{C}$ before gavage to mice.

\section{Animals and experimental design}


Seventy-two healthy male ICR mice (3-weeks-old) were obtained from the Experimental Animal Center of Shanxi Medical University (Taiyuan, Shanxi, China), where they were kept under specific pathogen-free (SPF) conditions. All mice were fed with sufficient food and water, and the environment was maintained at $22-25^{\circ} \mathrm{C}, 12 / 12-\mathrm{h}$ light/dark cycle. This animal study was approved by the Experimental Animal Ethics Committee of Shanxi Agricultural University (Taigu, Shanxi, China), and performed in accordance with their guidelines.

After one-week of acclimatization, the mice were randomly divided into nine groups $(n=8)$. In the blank control group (BC), mice were administered with $0.3 \mathrm{ml}$ saline intragastrically twice per day at 9 am and 1 $\mathrm{pm}$, respectively. In the ceftriaxone sodium group (CS, $4 \mathrm{~g} / \mathrm{kg} / \mathrm{d})$, mice were given $0.3 \mathrm{ml} \mathrm{CS}$ and saline by gavage at 9 am and $1 \mathrm{pm}$, respectively. For the CS + LP group (CS+LP), the CS + B group (CS+B), the CS + $S$ group $(C S+S)$, the $C S+S H$ group $(C S+S H)$, the $C S+$ Fermented $B$ group $(C S+F B)$, the $C S+$ Fermented $S$ group $(\mathrm{CS}+\mathrm{FS})$ and the Fermented $\mathrm{SH}$ group $(\mathrm{CS}+\mathrm{FSH})$, mice were given $0.3 \mathrm{ml} \mathrm{CS}$ at 9 am and the corresponding drug at $1 \mathrm{pm}$ by gavage, respectively, and treated for 7 days. On day 1, 4 and 7, diarrhea symptoms were recorded and fresh stool samples of mice in each group were taken for live bacterial culture. On day 8 , the mice were euthanized. The intestinal tissues were stored at $-80^{\circ} \mathrm{C}$ for qRT-PCR. Three samples of each group were fixed in $4 \%$ paraformaldehyde solution for intestinal histopathology.

\section{Diarrhea assessment and live bacterial culture}

All mice were kept separately with one mouse one cage. During the observation period of $2 \mathrm{~h}$, diarrhea symptoms were assessed by three indicators: fecal output weight[18], total number of fecal output[19], and fecal water content[18]. Fecal output was determined by measuring cumulative stool weight in $2 \mathrm{~h}$. Total number of fecal output in each group was obtained by counting the fecal numbers of mice. Fecal samples were weighed and dried, and then the dried solid weight and total fecal weight were measured. The fecal water content $=1-$ (dried solid weight $) /($ total fecal weight $)$.

Fresh feces were homogenized and serially diluted in saline solution. Aliquots were then spread onto different selective agar plates ( $10^{-2}$ to $10^{-7}$ dilutions). Lactobacillus Selective (LBS), Tryptone-yeast extract (TPY), Bile Esculin Azide (BEA) and Eosin-Methylene Blue (EMB) agar medium (Solarbio, Beijing, China) were used to detect lactobacillus, bifidobacterium, enterococcus and colibacillus, respectively. The plates were incubated at $37^{\circ} \mathrm{C}$ in an anaerobic or aerobic atmosphere for $48 \mathrm{~h}$.

\section{Intestinal morphometrics}

The duodenum, jejunum, and ileum tissues were embedded in paraffin and cut into $5 \mu \mathrm{m}$ sections and three slides of each sample were stained with hematoxylin \& eosin (HE) staining. Images were observed with an Olympus BX51 microscope equipped with CCD DP70 video camera (Olympus Optical, Tokyo, Japan). The villus height and crypt depth were measured using Image-Pro Plus (Version 5.1, Media Cybernetics, Silver Spring, MD, USA) and villus height to crypt depth ratio (VH/CD) was calculated.

\section{RNA extraction and qRT-PCR}


After $0.5-1 \mathrm{~cm}$ duodenum and colon tissues were cut into pieces, the pieces were added to $1 \mathrm{ml}$ Trizol for RNA extraction (Takara, Dalian, China). The quality of RNA was examined by NanoDrop ND-2000 Spectrophotometer (Nano-Drop, USA). Afterward, total RNA was reversely transcribed at $37^{\circ} \mathrm{C}$ for $15 \mathrm{~min}$, $85^{\circ} \mathrm{C}$ for 5 seconds and $4^{\circ} \mathrm{C}$ for 10 min using the PrimeScript ${ }^{\text {TM }}$ kit (Takara, Dalian, China). The sequences of reference gene and target gene, designed by Primer 3.0 plus, were as follows: GAPDH, AQP1, AQP3, AQP4, ZO-1, Occludin, and ZO-1 (Table 2). qRT-PCR was performed by Agilent Mx3000P QPCR (Stratagene, USA) using the SYBR ${ }^{\circledR}$ Premix Ex Taq ${ }^{\mathrm{TM}}$ II Kit (Takara, Dalian, China). qRT-PCR cycling conditions were $95^{\circ} \mathrm{C}$ for $30 \mathrm{~s}, 95^{\circ} \mathrm{C}$ for $5 \mathrm{~s}, 60^{\circ} \mathrm{C}$ (or $57^{\circ} \mathrm{C}$ ) for $30 \mathrm{~s}, 72{ }^{\circ} \mathrm{C}$ for $30 \mathrm{~s}, 95^{\circ} \mathrm{C}$ for $15 \mathrm{~s}, 60$ ${ }^{\circ} \mathrm{C}$ for $1 \mathrm{~min}, 95^{\circ} \mathrm{C}$ for $15 \mathrm{~s}$, and repeated for 40 (or 42) cycles. GAPDH was applied as an internal control, and the expression levels of the target gene were carried out with the $2^{-\Delta \Delta \mathrm{Ct}}$ method.

Table 2

Primer sequences for qRT-PCR

\begin{tabular}{|c|c|c|c|}
\hline Gene & Primer sequences $\left(5^{\prime} \rightarrow 3^{\prime}\right)$ & Product (bp) & Accession no. \\
\hline \multirow[t]{2}{*}{ GAPDH } & Forward: TGTGGATCAGCAAGCAGGAG & 87 & NM_001289726.1 \\
\hline & Reverse: ACGCAGCTCAGTAACAGTCC & & \\
\hline \multirow[t]{2}{*}{ AQP1 } & Forward: TTCCTGGTCTCAGAGCTTCC & 91 & NM_007472.2 \\
\hline & Reverse: TGGGTCCСТСАСТTTСАСТC & & \\
\hline \multirow[t]{2}{*}{ AQP3 } & Forward: CTTGTGATGTTTGGCTGTGG & 85 & NM_016689.2 \\
\hline & Reverse: AAGCCAAGTTGATGGTGAGG & & \\
\hline \multirow[t]{2}{*}{ AQP4 } & Forward: GTGTCTGTGGCAGCGAGATA & 116 & NM_001308641.1 \\
\hline & Reverse: GCATCTGCCTCAGAACATGA & & \\
\hline \multirow[t]{2}{*}{ AQP8 } & Forward: GGCTGAGAAGGTGGAGAATG & 135 & NM_001109045.1 \\
\hline & Reverse: AAAGAGGGTGTGGAGAGCAG & & \\
\hline \multirow[t]{2}{*}{ ZO-1 } & Forward: TTCAGCAGCAACAGAACCAG & 88 & XM_006540786.4 \\
\hline & Reverse: CGATCGTCATGCAAATCAAG & & \\
\hline \multirow[t]{2}{*}{ Occludin } & Forward: GAATGGCAAGCGATCATACC & 106 & NM_001360536.1 \\
\hline & Reverse: CTGCCTGAAGTCATCCACAC & & \\
\hline \multirow[t]{2}{*}{ Claudin-1 } & Forward: TGATCGCAATCTTTGTGTCC & 90 & NM_016674.4 \\
\hline & Reverse: CAATGACAGCCATCCACATC & & \\
\hline
\end{tabular}

\section{Identification of the compounds in S and FS}


The freeze-dried samples (six S and six FS) were crushed using a mixer mill (MM400, Retsch, Laichi, Germany) for $1.5 \mathrm{~min}$ at $30 \mathrm{~Hz} .100 \mathrm{mg}$ powder was weighted and extracted overnight at $4^{\circ} \mathrm{C}$ with $0.6 \mathrm{ml}$ $70 \%$ aqueous methanol. Following centrifugation at 10, $000 \mathrm{~g}$ for $10 \mathrm{~min}$, the extracts were absorbed and filtrated before UPLC-MS/MS analysis. Same volume was taken from each sample and pooled as Quality Control (QC) samples.

The sample extracts were analyzed using a 1290 UHPLC system (Agilent Technologies, La Jolla, CA, USA) combined with quadrupole time-of-flight mass spectrometer (QTOF) 6600 (AB Sciex, Redwood City, CA, USA). The chromatographic separations were performed on an Agilent Eclipse Plus C18 column (1.8 $\mu \mathrm{m}$, $2.1 \mathrm{~mm} * 100 \mathrm{~mm}$, Milford, MA, USA) with the column temperature set at $40^{\circ} \mathrm{C}$. The mobile phases were pure water with $0.04 \%$ acetic acid $(A)$ and acetonitrile with $0.04 \%$ acetic acid $(B)$. The gradient program was performed as follows: the starting conditions of $95 \% A, 5 \% B$, a linear gradient to $5 \% A, 95 \%$ within 10 min, a composition of $5 \%$ A, 95\% B kept for 1 min, and subsequently, a composition of $95 \% A, 5 \% B$ was adjusted within $0.10 \mathrm{~min}$ and kept for $2.9 \mathrm{~min}$. The injection volume was $4 \mu \mathrm{l}$.

According to Yang et al method[20], QTOF was operated using an Electron Spray lonization (ESI) in positive and negative ion mode and controlled by Analyst 1.6.3 software (AB Sciex, Waltham, MA, USA) to evaluate the full scan survey MS data. The ESI source operation parameters were as follows: source temperature $550{ }^{\circ} \mathrm{C}$; ion spray voltage (IS) $5500 \mathrm{~V}$ (positive ion mode)/-4500 V (negative ion mode); ion source gas I, gas II, and curtain gas were set at 50,60, and 30.0 psi, respectively.

\section{Statistical analysis}

All data were are represented as means $\pm S E M$. The significance of the difference between means was determined by analysis of variance (ANOVA) of Graph Pad Prism 6.0 (GraphPad Software Inc., San Diego, USA) followed by Tukey's test with $P<0.05$ being considered significant.

\section{Result}

\section{Disruption of the intestinal flora by antibiotics-triggered diarrhea in mice and alleviation of treatments}

In order to investigate the effects of TCM and fermented TCM (FTCM) on antibiotic-induced diarrhea, the related indexes were detected in $\mathrm{BC}, \mathrm{CS}, \mathrm{CS}+\mathrm{LP}, \mathrm{CS}+\mathrm{B}, \mathrm{CS}+\mathrm{S}, \mathrm{CS}+\mathrm{SH}, \mathrm{CS}+\mathrm{FB}, \mathrm{CS}+\mathrm{FS}$, and $\mathrm{CS}+\mathrm{FSH}$ groups, respectively. Fig. 1 represents the data of stool weigh, total number of fecal output and fecal water content in 2 hours. Compared to BC group, the CS group had a significant increase in fecal water content on day $1(P<0.05)$ (Fig. 1A1). Notably, on day 4 and 7, the CS group was significantly increased in stool weigh, total number of fecal output, and fecal water content compared to the BC group $(P<0.05)$ (Fig. 1B and $1 \mathrm{C}$ ), indicating the occurrence of diarrhea. Meanwhile, compared to CS group, the total number of fecal output was significantly decreased in seven groups $(P<0.05)$, and the fecal water content was a significant decrease in $\mathrm{CS}+\mathrm{B}, \mathrm{CS}+\mathrm{FB}, \mathrm{CS}+\mathrm{FS}$ and $\mathrm{CS}+\mathrm{FSH}$ groups (Fig. 1B2,3). As shown in Fig. 1C, the stool weight and fecal water content were significantly decreased in seven groups $(P<0.05)$, showing an 
alleviation by TCM, LP and FTCM. In CS+FS group, the stool weight, total number of fecal output and water content of fecal were remarkably lowed $(P<0.05)$ from day 4 to day 7 compared to CS group. But this did not happen in CS+S and CS+LP groups (Fig. 1B and 1C), which suggested FS has a continuous therapeutic effect.

Then, bacterial culture test was performed to examine whether above symptoms were accompanied with the disruption of some gut bacteria. The situation of live bacteria counting was presented in Fig. 2 . Compared to the BC group, the number of lactobacillus in the feces of the CS group showed a trend of significant decrease from day 1 to day $7(P<0.05)$. Besides the CS+LP group, there was no significant increase compared to CS group $(P>0.05)$ in other six treatment groups on day 7 (Fig. 2A). On day 1,4 and 7, the number of bifidobacterium in CS group was significantly lower than that in BC group $(P<0.05)$. Compared to the CS group, it was significantly increased in CS+LP, CS+S, CS+FS and CS+FSH groups on day $7(\mathrm{P}<0.05)$ (Fig. 2B). In addition, the number of enterococcus in CS group was significantly lower than that of $B C$ group on day 4 and $7(P<0.05)$. Slightly different from bifidobacterium, the number of enterococcus was significantly increased in CS+LP, CS+S, CS+FB and CS+FS groups compared to CS group on day 4 and $7(P<0.05)$ (Fig. $2 C$ ). In the $C S$ group, an obvious decrease in the abundance of colibacillus was observed on day 1,4 and $7(P<0.05)$, and besides $C S+B$ and $C S+S$ groups, the abundance had not been recovered on day 7 in other treatment groups.

\section{Treatments improved gut barrier function in dysbacteriosis mice induced by CS}

The small intestinal morphology was shown in Fig. 3, 4 and 5. It was observed that CS caused intestinal mucosal injury in duodenum, jejunum and ileum, including short and broad villi, deep crypts, incomplete and fractured structures. As seen in Fig. $3 \mathrm{~A}$ and $3 \mathrm{C}$, the villus height and $\mathrm{VH} / \mathrm{CD}$ of duodenum were reduced $(P<0.05)$ in CS group and the villus height was increased in CS+LP, CS+B, CS+S, CS+FB and $C S+F S$ groups $(P<0.05)$. Interestingly, the $V H / C D$ of duodenum was obviously increased only in $C S+S$ and $C S+F S$ groups $(P<0.05)$. Fig. 4 showed that mice with $C S$ had lower villus height and $V H / C D$ in jejunum than mice in $B C$ group $(P<0.05)$, and the crypt depth was enhanced notably in CS+LP group compared to $C S$ group $(P<0.05)$. The $V H / C D$ was increased significantly in $C S+S, C S+F B, C S+F S$ groups compared to $C S$ group $(P<0.05)$. Analogously, mice with $C S$ had shorter villus height and $V H / C D$ in ileum than mice in $\mathrm{BC}$ group $(\mathrm{P}<0.05)$ (Fig. $5 \mathrm{C})$. Unlike jejunum, the villus height of ileum enhanced in $\mathrm{CS}+\mathrm{SH}$ group, and the $\mathrm{VH} / \mathrm{CD}$ greatly enhanced in $\mathrm{CS}+\mathrm{S}, \mathrm{CS}+\mathrm{SH}$ and $\mathrm{CS}+\mathrm{FB}$ groups $(\mathrm{P}<0.05)$, respectively.

The mRNA relative expression of aquaporins (AQP1, 3, and 4) and TJ proteins (ZO-1 and Occludin) in duodenum and colon are presented in Fig. 6 and 7. Compared to BC group, the mRNA expression levels of AQP1 and ZO-1 were markedly reduced $(P<0.05)$ in CS gavaged mice. However, the AQP1 mRNA expressions was enhanced in the $C S+L P, C S+B$ and $C S+F S$ groups as compared to the $C S$ group $(P<$ 0.05). And the ZO-1 expression was obviously increased in $C S+L P, C S+B$ and $C S+S$ groups as compared to the CS group $(P<0.05)$. Furthermore, although compared to BC group, the mRNA expressions of AQP4 
was not significantly decreased in CS group, when compared to the CS group, it was obviously enhanced in $\mathrm{CS}+\mathrm{FS}$ group $(\mathrm{P}<0.05)$ (Fig. 6).

As seen in Fig. 7, the mRNA expression levels of AQP1 and ZO-1 were evidently increased $(P<0.05)$ in other treatment groups as compared to CS group. In addition, the Occludin mRNA was obviously higher in $\mathrm{CS}+\mathrm{B}, \mathrm{CS}+\mathrm{S}, \mathrm{CS}+\mathrm{SH}$ and $\mathrm{CS}+\mathrm{FS}$ groups than $\mathrm{CS}$ group $(\mathrm{P}<0.05)$. Whereas, compared with the $\mathrm{BC}$ group, the changes in AQP3 and AQP4 mRNA expression were not significant in other treatment groups.

\section{Identification of the compounds in S and FS}

A principal component analysis (PCA) model was constructed in Fig. 8A. The aggregation trend of two groups was absolutely separated in this model, and the spots were gathered together in their own group. To investigate distinct significant compounds between $S$ and FS, the orthogonal partial least squaresdiscriminant analysis (OPLS-DA) model was established. An obvious separation trend between the two groups was observed in the Fig. $8 \mathrm{~B}$, and the interpretation rate of the model to $X$ and $Y$ matrix parameters $\mathrm{R} 2 \mathrm{X}=0.809(80.9 \%), \mathrm{R} 2 \mathrm{Y}=1(100 \%)$, and prediction ability Q2Y $=0.997(99.7 \%)$ indicated that the model had an excellent reliability and prediction.

The results of heat map (Fig. 9A) showed the distribution of differential compounds in the $S$ and FS groups. Differential compounds were screened out $(\mathrm{FC}>1.2$, P value $<0.05$ and VIP $>1)$, and 121 significantly changed compounds were identified in volcano plot (Fig. 9B). Among them, 30 compounds in the FS group were obviously up-regulated compared to the $S$ group, such as Pyrocatechol, Nicotinic acid and (S)-(-)-2-Hydroxyisocaproic acid, etc, and 91 compounds were significantly down-regulated, like Maleic acid, Nicotinamide, Poricoic acid etc. The details were shown in supplementary file 1.

The Kyoto Encyclopedia of Genes and Genomes (KEGG) classification results were shown in Fig. 10. Six pathways were annotated that more than four significantly different compounds were involved in: Biosynthesis of secondary metabolites (11), Metabolic pathways (26), 2-0xocar boxylic acid metabolism (4), Biosynthesis of amino acids (4), Pyrimidine metabolism (5), Purine metabolism (6). The details were shown in supplementary file 2 .

\section{Discussion}

The gut microbiota is a complex ecosystem that provides essential functions, including carbohydrate metabolism, interaction with immune system, and prevention against pathogen invasion[21]. The intestinal flora is susceptible to dysbacteriosis caused by external and internal factors, and antibiotic is one of the main causes[22]. To observe the alleviation of TCM, LP, and FTCM on dysbacteriosis, we established a mouse dysbacteriosis model by intragastric administration of CS. Ceftriaxone is a broad spectrum, third-generation cephalosporin that is widely used to treat gastrointestinal infections. Repeated overuse of this antibiotic can disrupt the equilibrium of intestinal flora and cause side effects such as antibiotic-associated diarrhea[23]. In the present study, there are obvious diarrhea symptoms induced by CS, including increased stool weigh, total number of fecal output and fecal water content. Then, bacterial 
culture tests showed that there was a destruction of some intestinal flora in mice gavaged antibiotic, which is similar to Zeng's research[24]. Overall, the above results are consistent with our previous studies that ceftriaxone and ciprofloxacin can cause watery diarrhea and intestinal microbial disorders in animal models[25].

An increasing body of evidence showed that probiotics can not only inhibit the proliferation of harmful bacteria in the intestine, promote the proliferation of beneficial bacteria, effectively restore and balance the intestinal flora, but the intestinal flora also participates in immune regulation and enhances immunity, which help prevent and treatment of antibiotic-induced diarrhea[26]. Notably, some studies found that the proportion of spleen deficiency type is the largest among antibiotic-associated diarrhea patients, and various tonifying Qi-invigorating TCMs are used to cure bowel diseases, treat intestinal flora disorder, enhance immunity, relieve fatigue, and prolong lifespan either as clinical medications or daily diets[8]. Qiweibaizhu powder could effectively treat dysbiosis diarrhea by improving intestinal flora and promoting the reproduction of beneficial bacteria such as bifidobacteria and lactobacillius[24, 27]. Therefore, probiotics combined with TCM may regulate balance in gut microbiota to reduce dysbiosis. In this study, the water content of the above animal feces was significantly reduced after continuous treatment with TCM, LP, and FTCM for 7 days, among which, FS significantly constantly relieved diarrhea symptoms from day 4 to day 7 , and on day 7 , compared to $S$, the fecal water content of mice was obviously decreased in FS. The number of lactobacillus and bifidobacterium of mice in LP, FS and FSH increased significantly compared to the CS group level, and the number of colibacillus decreased significantly, which shows that LP, FS and FSH have the function of supporting the growth of beneficial flora and clearing intestinal pathogenic bacteria. The reason may be LP fermentation in S and SH altered the gut $\mathrm{pH}$ that inhibit the proliferation of colibacillus[28]. Although B and S increased the number of beneficial bacteria, they also promoted the growth of harmful bacteria (colibacillus), showing that fermentation has certain advantages. The effect of $\mathrm{SH}$ is unstable and may be related to its composition, which needs further study.

The intestine is one of the important visceral organs, not only for digestion and absorption of nutrients but also for its innate barrier protecting the body from pathogenic microorganisms. Small intestine tissue can be observed by H\&E staining. The serious damage to the small intestine chorionic villi and the histomorphological changes suggest that the small intestine absorption function and barrier function is severely damaged[29]. Studies have shown that Chinese medicine has obvious advantages in the treatment of intestinal mucosal barrier dysfunction[30]. He et al. treated broilers with probiotic (Bacillus subtilis, Bacillus licheniformis, and Saccharomyces cerevisiae), which increased VH/CD in duodenum, as well as higher ZO-1 mRNA expression in jejunum[31]. In addition, piglets treated with $\angle P 299 v$ had a lower diarrhea incidence than the control, the $\mathrm{VH} / \mathrm{CD}$ and ZO-1 mRNA level in the jejunum and ileum were increased, and the structure of the gut microbiota were modified[32]. After diarrhea in mice, the water in the intestine will increase, then promote the softening of feces, and stimulate the intestinal mucosa to a certain extent, thus causing mucosal damage. In the present study, the villus height of duodenum was increased after mice were fed with LP, B, S, FB and FS, and the VH/CD of duodenum was obviously increased by gavage with $\mathrm{S}$ and $\mathrm{FS}$, which is similar to above researches. The $\mathrm{VH} / \mathrm{CD}$ of jejunum was 
enhanced in $\mathrm{CS}+\mathrm{S}, \mathrm{CS}+\mathrm{FB}, \mathrm{CS}+\mathrm{FS}$ groups. SH improved the villus height and $\mathrm{VH} / \mathrm{CD}$ of ileum. Overall, the above treatments could promote the growth of the intestine, and reduce the stimulation of diarrhea on the intestinal mucosa and epithelium microvilli.

Furthermore, intestinal epithelial cells are connected through tight junctions (TJ), which regulates the intestinal barrier permeability and epithelial integrity. Therefore, the homeostasis expression of TJ protein is essential for the maintenance of human health[33]. ZO-1 and Occludin are the most critical components in the structural and functional organization of TJ[34]. The gut flora target various intracellular pathways, alter the expression and distribution of TJ proteins, then regulate intestinal barrier function[35]. Aquaporins (AQPs) are water-channel membrane proteins expressed in various tissues. Reportedly, at least 7 AQP subtypes (AQPs 1, 3, 4, 5, 7, 8, 9, and 11) are expressed in the gastrointestinal tract and play important roles in several physiological and pathological processes[36]. In particular, the distal small intestine and proximal colon are the major sites for AQP1, 3, and 4 expression[37]. Zhang et al.[18] observed that the antibiotic-associated diarrhea rats exhibited defective gastrointestinal integrity and improper epithelial organization, with decreased expression of aquaporin-encoding genes, aberrant TJ proteins, and a decrease in the number of goblet cells compared with control animals. Likewise, we found that the AQP1 and ZO-1 mRNA expression levels in the duodenum and colon were significantly attenuated in diarrhea mice. And the two gene expression levels were enhanced in mice of giving LP, $B, S$ or FS. These results are roughly in accordance with the results observed for the intestinal morphology, suggesting that TCM, probiotic, and FTCM display beneficial properties at the molecular level.

Since FS showed better advantages in most indicators, compounds in S and FS were detected by UHPLCQ-TOF/MS. Results showed that 30 compounds in FS were obviously up-regulated compared to S. In which, the four compounds were noteworthy of attention, including (S)-(-)-2-Hydroxyisocaproic acid, LMethionine, 4-Guanidinobutyric acid (4GBA), and Phenyllactate (PLA). (S)-(-)-2-Hydroxyisocaproic acid also named L-Leucine, and it is a signaling amino acid in animal metabolism, which can elevate villus height of duodenum and VH/CD of duodenum and ileum[38]. That is similar to our results. L-Leucine as an up-regulated compound in FS, indicating it could improve intestinal development. In addition, LMethionine is an essential amino acid (AA) in humans and other vertebrates. It can't be synthesized by the body and must be obtained from the diet. Methionine absorption from the gastrointestinal tract is highly efficient[39]. Studies have shown that dietary supplementation of methionine is beneficial to intestinal development and antioxidant function of pigs[40]. The two AA may play a significant role in improving intestinal health of diarrhea mice. 4GBA is an alkaloid included in guanidino compounds, and inhibits the growth of $H$. pylori in a dose dependent manner and might be useful in the treatment and/or protection of gastritis[41]. With the same functional group, 4-methylguanidine butyric acid inhibits bacteria and fungi, such as Staphylococcu saureus, Escherichia coli, Saccharomyces cerevisiae and Aspergillus niger[42]. In this experiment, the growth of colibacillus was inhibited in mice that gavaged FS, but further studies are needed to investigate whether it related to the increase of 4GBA. PLA is found in various foods, such as honey, pickle, sourdough and a variety of fermented foods[43]. It has versatile antimicrobial activity against food-borne pathogenic bacteria[44] and spoilage mould[45]. PLA has great potential for applications in the food, feed and pharmaceutical. Therefore, increased PLA in FS have an 
important antibacterial effect possibly. Perhaps the four compounds can be potential indicators of inhibiting harmful bacteria and improving intestinal development.

\section{Conclusion}

Taken together, $4 \mathrm{~g} / \mathrm{kg}$ CS altered the normal gut flora, caused diarrhea, induced short and broad villi, incomplete and fractured intestinal structures, and the decreased mRNA expressions related to intestinal barrier function. Meanwhile, TCM, LP, and FTCM all alleviated the alterations induced by CS in different degrees, especially, FTCM showed more sustained mitigative effects. Collectively, these findings provide a convincing experimental evidence and theoretical basis for further development and application of TCM, probiotic and FTCM in the clinical use of antibiotics.

\section{Abbreviations}

B: Buzhongyiqi decoction, S: Sijunzi decoction, SH: Shenlingbaizhu decoction, TCM: Traditional Chinese Medicine, FTCM: fermented Traditional Chinese Medicine, LP: Lactobacillus plantarum, AAD: antibioticassociated diarrhea, FS: Fermented S, CS: Ceftriaxone Sodium, UHPLC-Q-TOF/MS: ultra-high-performance liquid chromatography coupled with quadrupole time-of-flight mass spectrometry, AQPs: Aquaporins, TJ: tight junction, PCA: principal component analysis, OPLS-DA: orthogonal partial least squares-discriminant analysis, KEGG: Kyoto Encyclopedia of Genes and Genomes, 4GBA: 4-Guanidinobutyric acid, PLA: Phenyllactate.

\section{Declarations}

\section{Acknowledgments}

Not applicable.

\section{Author contributions}

Xin Guo: Methodology, Formal analysis, Investigation, Writing-Original Draft Preparation; Zipeng Yan: Data curation; Jixiang Wang: Data curation; Xinfeng Fan: Project administration; Jie Kang: Validation, Methodology; Ruiyan Niu: Project administration; Zilong Sun: Conceptualization, Resources, WritingReview \& Editing Preparation, Funding acquisition. All authors reviewed the manuscript.

\section{Funding}

This research was supported by the Program for Natural Science Foundation of Shanxi (Grant No. 201701D121102) and the Horizontal Cooperation Project of Shanxi Agricultural University (Grant No. 2018HX07).

\section{Ethics approval and consent to participate}


All institutional and national guidelines for the care and use of laboratory animals were followed.

\section{Availability of data and materials}

The datasets used and/or analysed during the current study are available from the corresponding author on reasonable request.

\section{Consent for publication}

Not applicable.

\section{Competing interests}

The authors declare that they have no competing interests.

\section{References}

1. Kim S, Covington A, Pamer EG. The intestinal microbiota: antibiotics, colonization resistance, and enteric pathogens. Immunol. Rev. 2017; 279 (1): 90-105.

2. Loho T, Dharmayanti A. Colistin: an antibiotic and its role in multiresistant Gram-negative infections. Acta Med. Indones. 2015; 47 (2):157-168.

3. Stahlmann R, Lode H. Safety considerations of fluoroquinolones in the elderly: an update. Drugs Aging 2010; 27 (3): 193-209.

4. Neuman $\mathrm{H}$, Forsythe $\mathrm{P}$, Uzan A, Avni O, Koren O. Antibiotics in early life: dysbiosis and the damage done. FEMS Microbiol. Rev. 2018; 42 (4): 489-499.

5. Jandhyala SM, Talukdar R, Subramanyam C, Vuyyuru H, Sasikala M, Reddy DN. Role of the normal gut microbiota. World J. Gastroenterol. 2015; 21 (29): 8787-8803.

6. Patel R, DuPont HL. New approaches for bacteriotherapy: prebiotics, new-generation probiotics, and synbiotics. Clin. Infect. Dis. 2015; 60 (Suppl 2): S108-S121.

7. Feng WW, Ao H, Peng C, Yan D. Gut microbiota, a new frontier to understand traditional Chinese medicines. Pharmacol. Res. 2019; 142: 176-191.

8. Wang XM, Li XB, Peng Y. Impact of Qi-invigorating traditional Chinese medicines on intestinal flora: A basis for rational choice of prebiotics. Chin. J. Nat. Med. 2017; 15 (4): 241-254.

9. Chen F, Wen Q, Jiang J, Li HL, Tan YF, Li YH, Zeng NK. Could the gut microbiota reconcile the oral bioavailability conundrum of traditional herbs? J. Ethnopharmacol. 2016; 179: 253-264.

10. Zu XP, Nagle DG, Zhou YD, Zhang WD. Application of intestinal flora in the study of TCM formulae, In: Zhang, W.D. (Ed.), Systems biology and its application in TCM formulas research, Academic Press, 2018, pp. 97-112. ISBN: 978-0-12-812744-5, Available from: https://doi.org/10.1016/B978-0-12812744-5.00005-9 
11. Xu C, Ji GE. Bioconversion of flavones during fermentation in milk containing Scutellaria baicalensis extract by Lactobacillus brevis. J Microbiol Biotechnol. 2013;23 (10): 1422-1427.

12. Wang J, Bose S, Kim H, Han K, Kim H. Fermented Rhizoma Atractylodis Macrocephalae alleviates high fat diet-induced obesity in association with regulation of intestinal permeability and microbiota in rats. Sci Rep. 2015; 5: 8391.

13. Oh J, Jeon SB, Lee Y, Lee H, Kim J, Kwon BR, Yu KY, Cha JD, Hwang SM, Choi KM, Jeong YS. Fermented red ginseng extract inhibits cancer cell proliferation and viability. J Med Food. 2015; 18 (4): 421-428.

14. Guo R, Guo S, Gao X, Wang H, Hu W, Duan R, Dong TTX, Tsim KWK. Fermentation of Danggui Buxue Tang, an ancient Chinese herbal mixture, together with Lactobacillus plantarum enhances the antidiabetic functions of herbal product. Chin. Med. 2020; 15 (1): 98.

15. Yim NH, Gu MJ, Park HR, Hwang YH and Ma JY, Enhancement of neuroprotective activity of Sagunjatang by fermentation with lactobacillus strains. BMC Complem. Altern. Med. 2018; 18: 312.

16. Yan Y, Du CH, Li ZY, Zhang M, Li J, Jia JP, Li AP, Qin XM, Qiang Song. Comparing the antidiabetic effects and chemical profiles of raw and fermented Chinese Ge-Gen-Qin-Lian decoction by integrating untargeted metabolomics and targeted analysis. Chin. Med. 2018; 13: 54.

17. Liu Y, Yang JS, Cai ZW. Chemical investigation on Sijunzi decoction and its two major herbs Panax ginseng and glycyrrhiza uralensis by LC/MS/MS. J. Pharmaceut. Biomed. Anal. 2006; 41 (5): 16421647.

18. Zhang WD, Zhu B, Xu JH, Liu YY, Qiu EQ, Li ZJ, Li ZC, He Y, Zhou HW, Bai Y, Zhi FC. Bacteroides fragilis protects against antibiotic-associated diarrhea in rats by modulating intestinal defenses. Front. Immunol. 2018; 9: 1040.

19. Umer S, Tekewe A, Kebede N. Antidiarrhoeal and antimicrobial activity of Calpurnia aurea leaf extract. BMC Complem. Altern. Med. 2012; 13: 21.

20. Yang W, Su Y, Dong GQ, Qian GT, Shi YH, Mi YL, Zhang YM, Xue JP, Du W, Shi TX, Chen SL, Zhang Y, Chen QF, Sun W. Liquid chromatography-mass spectrometry-based metabolomics analysis of flavonoids and anthraquinones in Fagopyrum tataricum L. Gaertn. (tartary buckwheat) seeds to trace morphological variations. Food Chem. 2020; 331: 127354.

21. Marques TM, Holster S, Wall R, König J, Brummer RJ. Correlating the gut microbiome to health and disease, In: Hyland, N., and Stanton, C. (Eds.), The Gut-Brain Axis, Academic Press, 2016, pp. 261-291. ISBN: 978-0-12-802304-4, Available from: http://dx.doi.org/10.1016/B978-0-12-802304-4.00012-8

22. Kabbani TA, Pallav K, Dowd SE, Galvez JV, Vanga RR, Castillo NE, Hansen J, Dennis M, Leffler DA, Kelly CP. Prospective randomized controlled study on the effects of Saccharomyces boulardii CNCM I-745 and amoxicillin-clavulanate or the combination on the gut microbiota of healthy volunteers. Gut Microbes 2017; 8 (1): 17-32.

23. Li M, Li WH, Wen S, Liu YH, Li T. Effects of ceftriaxone-induced intestinal dysbacteriosis on dendritic cells of small intestine in mice. Microbiol. Immunol. 2013; 57 (8): 561-568. 
24. Zeng A, Zhang HL,Tan ZJ, Cai Y, Cai GX, Zhou SN. The construction of mice diarrhea model due to dysbacteriosis and curative effect of ultra-micro Qiweibaizhusan. Microbiol. China 2012; 39: 13411348. (in Chinese)

25. Wang J, Guo X, Li R, Wang JX, Wang XW, Sun ZL. Effects of ceftriaxone sodium and ciprofloxacin on intestinal flora in mice. Prog. Vet. Med. 2020; 41 (1): 66-70. (in Chinese)

26. Tian P, Xu B, Sun HX, Li XY, Li Z, Wei PJ. Isolation and gut microbiota modulation of antibioticresistant probiotics from human feces. Diagn. Microbiol. Infect. Dis. 2014; 79 (4): 405-412.

27. Guo KX, Tan ZJ, Xie MZ, She Y, Wang XH. The synergic effect of ultra-micro powder Qiweibaizhusan combined with yeast on dysbacteriotic diarrhea mice. Chin. J. Appl. Environ. Biol. 2015; 21 (1): 61-67. (in Chinese)

28. Che LQ, Xu Q, Wu C, Luo YH, Huang XB, Zhang B, Auclair E, Kiros T, Fang ZF, Lin Y, Xu SY, Feng B, Li J, Wu D. Effects of dietary live yeast supplementation on growth performance, diarrhoea severity, intestinal permeability and immunological parameters of weaned piglets challenged with enterotoxigenic Escherichia coli K88. Brit. J. Nutr. 2017; 118 (11): 949-958.

29. Chen Z, Xie J, Hu MY, Tang J, Shao ZF, Li MH. Effect of Y-aminobutyric acid on digestive enzymes, absorption function, and immune function of intestinal mucosa in heat-stressed chicken. Poultry Sci. 2015; 93 (10): 2490-2500.

30. Long CX, Shao HQ, Luo CY, Yu R, Tan ZJ. Bacterial diversity in the intestinal mucosa of dysbiosis diarrhea mice treated with Qiweibaizhu powder. Gastroent. Res. Pract. 2020; 2020: 9420129.

31. He TF, Long SF, Mahfuz S, Wu D, Wang X, Wei XM, Piao XS. Effects of probiotics as antibiotics substitutes on growth performance, serum biochemical parameters, intestinal morphology, and barrier function of broilers. Animals Basel 2019; 9 (11): 985.

32. Wang Q, Sun Q, Qi RL, Wang J, Qiu XY, Liu ZH, Huang JX. Effects of Lactobacillus plantarum on the intestinal morphology, intestinal barrier function and microbiota composition of suckling piglets. J. Anim. Physiol. Anim. Nutr. 2019; 103 (6) : 1908-1918.

33. Robinson K, Deng Z, Hou YQ, Zhang GL. Regulation of the intestinal barrier function by host defense peptides. Front. Vet. Sci. 2015; 2 (6): 57.

34. Suzuki T. Regulation of intestinal epithelial permeability by tight junctions. Cell. Mol. Life Sci. 2013; 70 (4): 631-659.

35. Ulluwishewa D, Anderson RC, McNabb WC, Moughan PJ, Wells JM, Roy NC. Regulation of tight junction permeability by intestinal bacteria and dietary components. J. Nutr. 2011; 141 (5): 769-776.

36. King LS, Kozono D, Agre P. From structure to disease: the evolving tale of aquaporin biology. Nat. Rev. Mol. Cell Bio. 2004; 5 (9): 687-698.

37. Cao MW, Yang M, Ou ZY, Li DY, Geng LL, Chen PY, Chen H, Gong ST. Involvement of aquaporins in a mouse model of rotavirus diarrhea. Virologica Sinica 2014; 29: 211-217.

38. Sun YL, Wu ZL, Li W, Zhang C, Sun KJ, Ji Y, Wang B, Jiao N, He BB, Wang WW, Dai ZL, Wu GY. Dietary L-leucine supplementation enhances intestinal development in suckling piglets. Amino Acids 2015; 47 (8): 1517-1525. 
39. Mastrototaro L, Sponder G, Saremi B, Aschenbach JR. Gastrointestinal methionine shuttle: priority handling of precious goods. IUBMB Life 2016; 68 (12): 924-934.

40. Su WP, Zhang H, Ying ZX, Li Y, Zhang LG, Zhang LL, Wang T. Effect of dietary methionine supplementation on intestinal development and antioxidant function in growing pigs with intrauterine growth retardation. Food Sci. 2018; 39: 132-138. (in Chinese)

41. Hwang IY, Jeong CS. Inhibitory effects of 4-Guanidinobutyric acid against gastric lesions. Biomol. Ther. 2012; 20 (2): 239-244.

42. Guo R, Zhao YY, Wei P, Lai X, Sun J, Guo YX, Long XJ. Synthesis, characterization and bacterization and bacteriostatic activity of 4-methylguanidine butyric acid. Sci. Technol. Food Ind. 2011; 32 (1): 8789. (in Chinese)

43. Sun DQ, Li HF, Song DW, Zhang LP, Zhao X, Xu XX. Genome, transcriptome and fermentation analyses of Lactobacillus plantarum LY-78 provide new insights into the mechanism of phenyllactate biosynthesis in lactic acid bacteria. Biochem. Biophys. Res. Commun. 2019; 519 (2): 351-357.

44. Ning YW, Yan AH, Yang K, Wang ZX, Li XF, Jia YM. Antibacterial activity of phenyllactic acid against Listeria monocytogenes and Escherichia coli by dual mechanisms. Food Chem. 2017; 228: 533-540.

45. Crowley S, Mahony J, Sinderen DV. Current perspectives on antifungal lactic acid bacteria as natural bio-preservatives. Trends Food. Sci. Tech. 2013; 33 (2): 93-109.

\section{Figures}



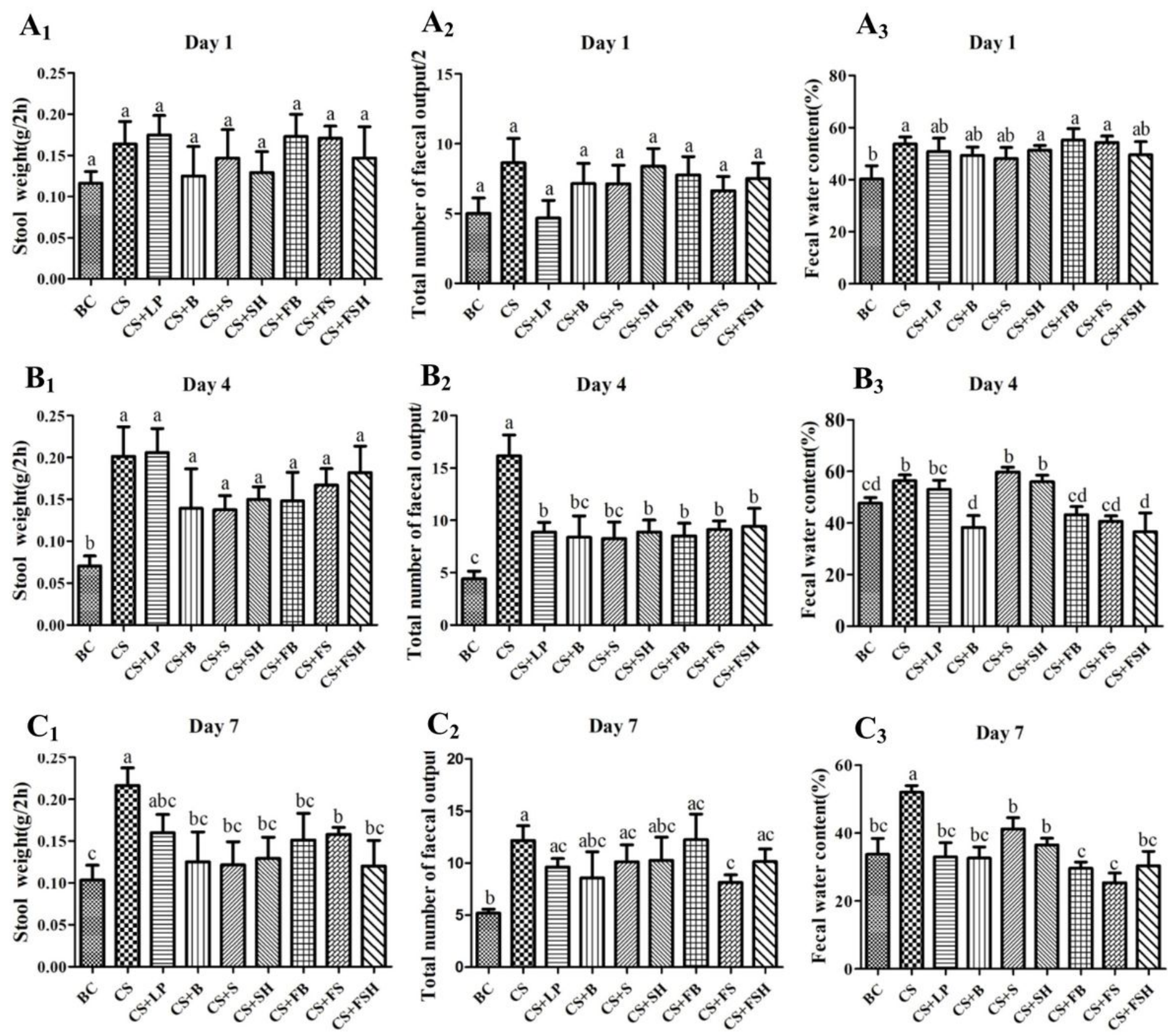

Figure 1

Stool weigh, total number of fecal output and fecal water content in 2 hours of day $1(A), 4$ (B) and 7(C). BC: Blank Control, CS: Ceftriaxone Sodium, CS+LP: Ceftriaxone Sodium + Lactobacillus Plantarum, CS+B: Ceftriaxone Sodium + Buzhongyiqi decoction, CS+S: Ceftriaxone Sodium + Sijunzi decoction, CS+SH: Ceftriaxone Sodium + Shenlingbaizhu decoction, CS+FB: Ceftriaxone Sodium + Fermented Buzhongyiqi decoction, CS+FS: Ceftriaxone Sodium + Fermented Sijunzi decoction and CS+FSH: Ceftriaxone Sodium + Fermented Shenlingbaizhu decoction. $n=8$. Mean values with unlike letters were significantly different $(P<0.05)$. 

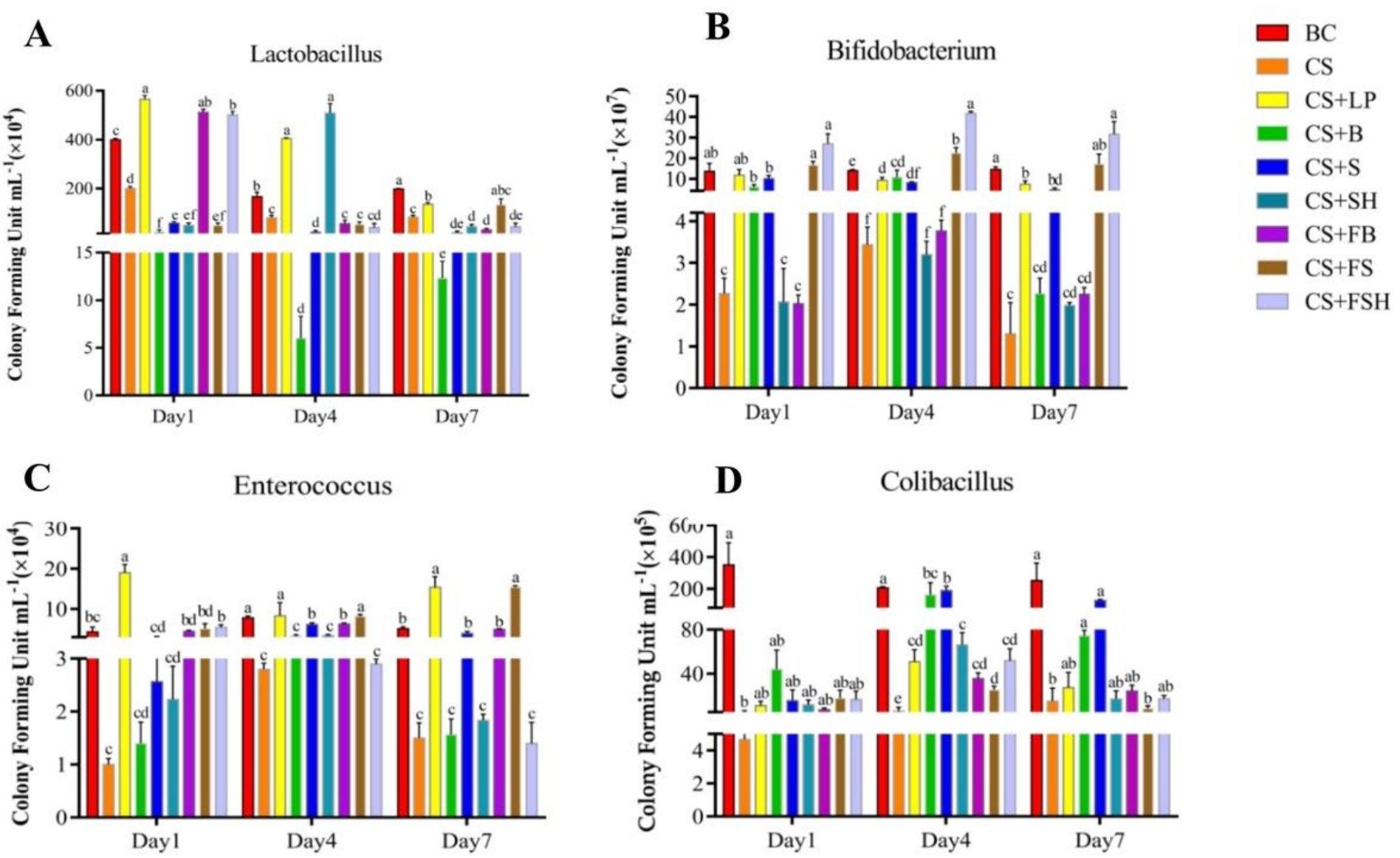

Figure 2

Live bacteria counting of lactobacillus (A), bifidobacterium (B), enterococcus (C) and colibacillus (D) on day 1, 4 and 7 of treatments. BC: Blank Control, CS: Ceftriaxone Sodium, CS+LP: Ceftriaxone Sodium + Lactobacillus Plantarum, CS+B: Ceftriaxone Sodium + Buzhongyiqi decoction, CS+S: Ceftriaxone Sodium + Sijunzi decoction, CS+SH: Ceftriaxone Sodium + Shenlingbaizhu decoction, CS+FB: Ceftriaxone Sodium + Fermented Buzhongyiqi decoction, CS+FS: Ceftriaxone Sodium + Fermented Sijunzi decoction and $\mathrm{CS}+\mathrm{FSH}$ : Ceftriaxone Sodium + Fermented Shenlingbaizhu decoction. $\mathrm{n}=3$. Mean values with unlike letters were significantly different $(P<0.05)$. 

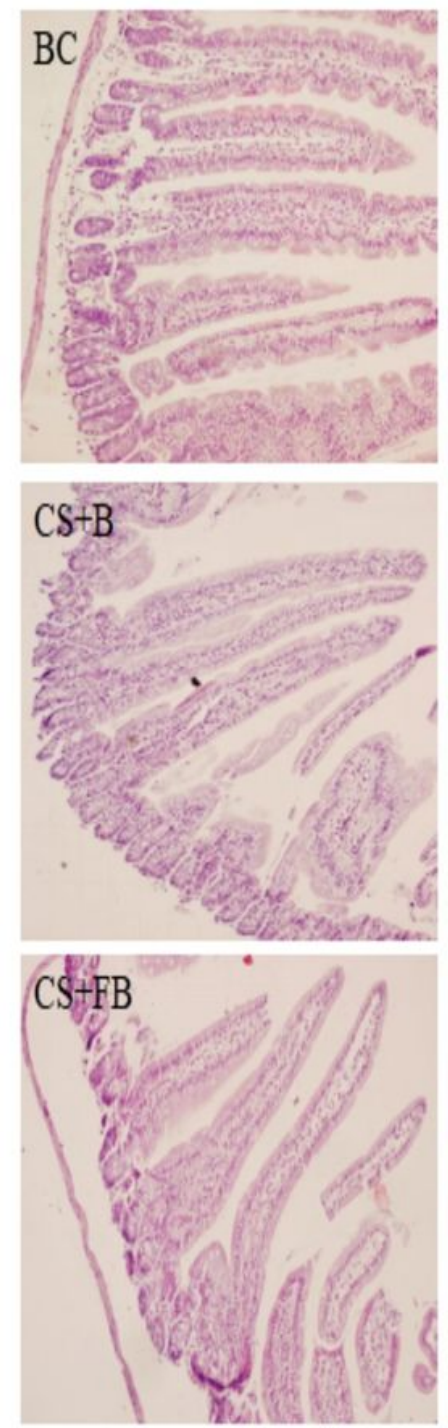
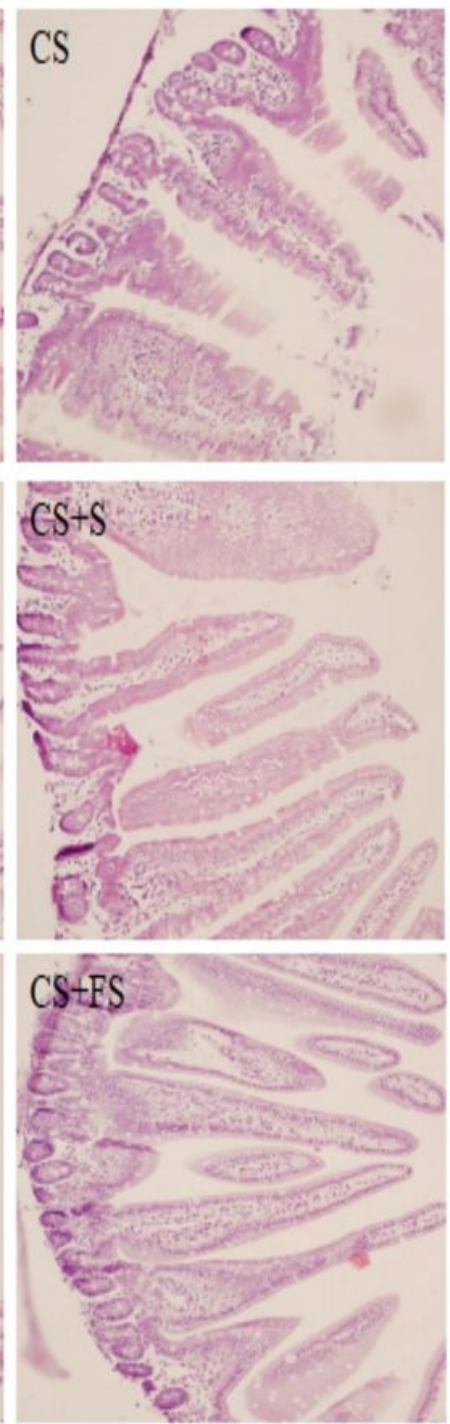
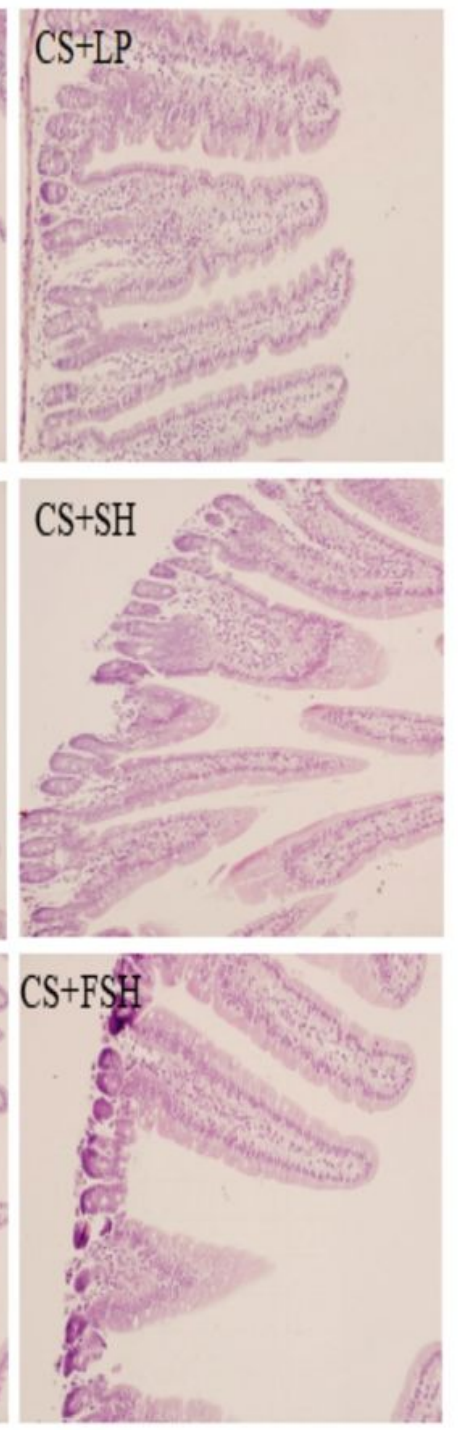

A
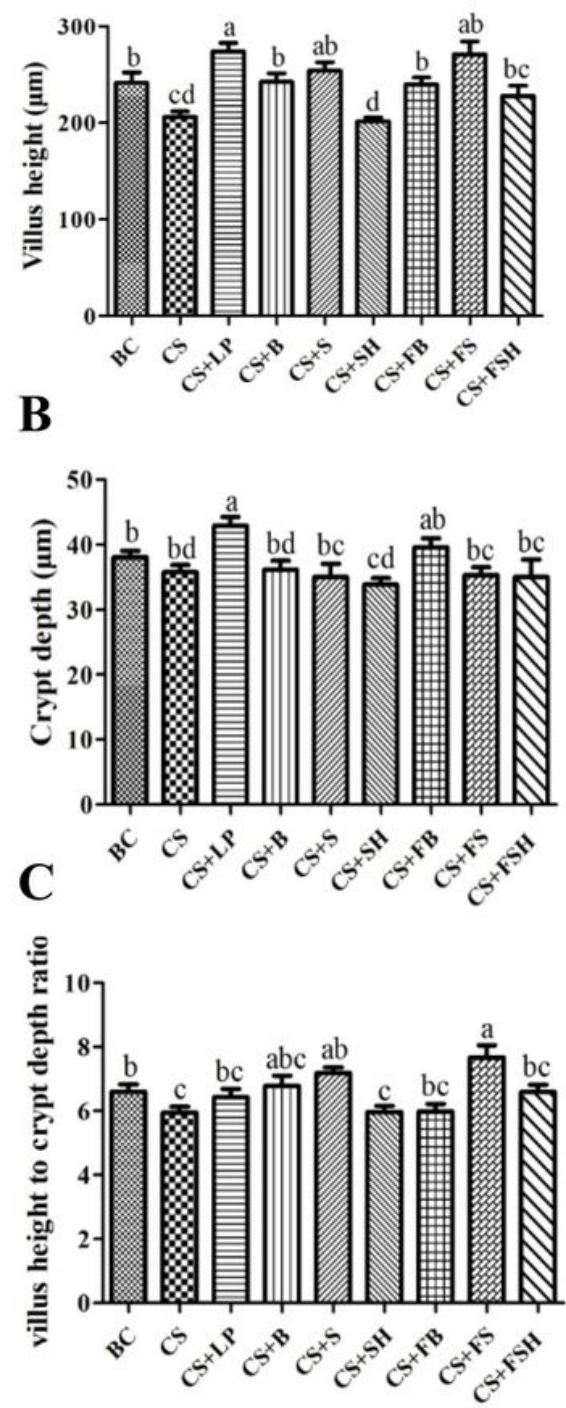

\section{Figure 3}

Intestinal morphology (200x) of duodenum in mice with dysbacteriosis induced by ceftriaxone sodium and the alleviation of treatments. A: villus height, B: crypt depth, C: villus height to crypt depth ratio (VH/CD). BC: Blank Control, CS: Ceftriaxone Sodium, CS+LP: Ceftriaxone Sodium + Lactobacillus Plantarum, CS+B: Ceftriaxone Sodium + Buzhongyiqi decoction, CS+S: Ceftriaxone Sodium + Sijunzi decoction, CS+SH: Ceftriaxone Sodium + Shenlingbaizhu decoction, CS+FB: Ceftriaxone Sodium + Fermented Buzhongyiqi decoction, CS+FS: Ceftriaxone Sodium + Fermented Sijunzi decoction and $\mathrm{CS}+\mathrm{FSH}$ : Ceftriaxone Sodium + Fermented Shenlingbaizhu decoction. $\mathrm{n}=3$. Mean values with unlike letters were significantly different $(P<0.05)$. 

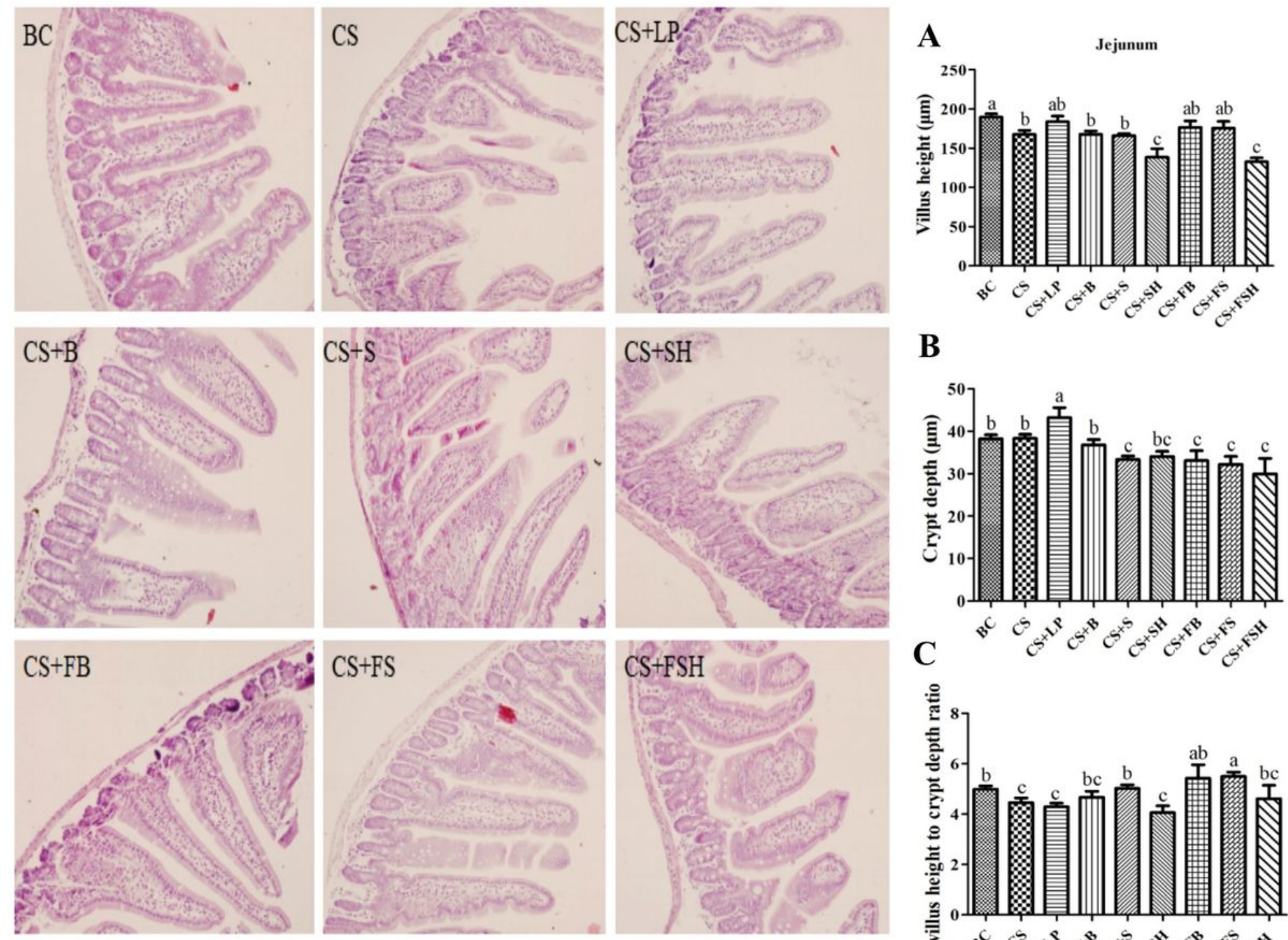

B
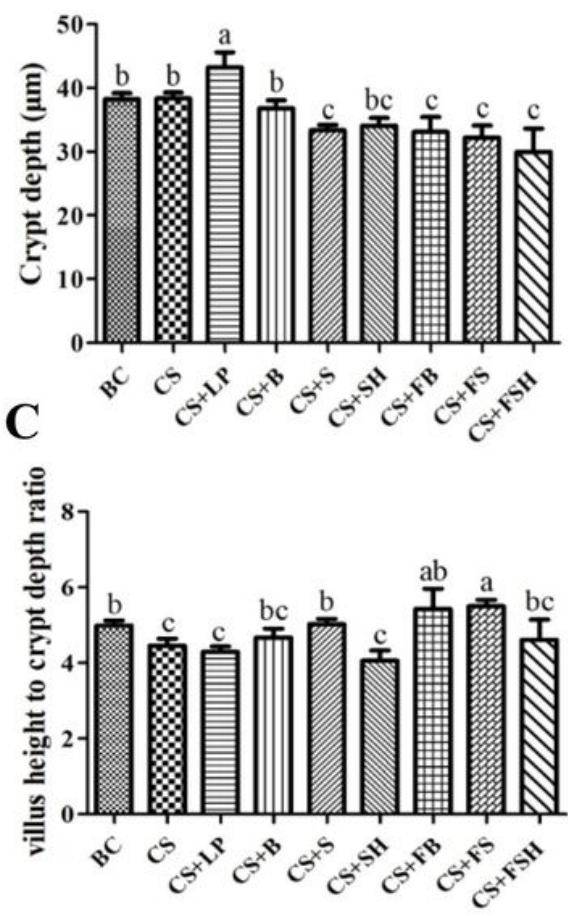

Figure 4

Intestinal morphology (200x) of jejunum in mice with dysbacteriosis induced by ceftriaxone sodium and the alleviation of treatments. A: villus height, $B$ : crypt depth, C: villus height to crypt depth ratio $(\mathrm{VH} / \mathrm{CD})$. BC: Blank Control, CS: Ceftriaxone Sodium, CS+LP: Ceftriaxone Sodium + Lactobacillus Plantarum, CS+B: Ceftriaxone Sodium + Buzhongyiqi decoction, $\mathrm{CS}+\mathrm{S}$ : Ceftriaxone Sodium + Sijunzi decoction, $\mathrm{CS}+\mathrm{SH}$ : Ceftriaxone Sodium + Shenlingbaizhu decoction, CS+FB: Ceftriaxone Sodium + Fermented Buzhongyiqi decoction, CS+FS: Ceftriaxone Sodium + Fermented Sijunzi decoction and CS+FSH: Ceftriaxone Sodium + Fermented Shenlingbaizhu decoction. $n=3$. Mean values with unlike letters were significantly different $(P<0.05)$. 

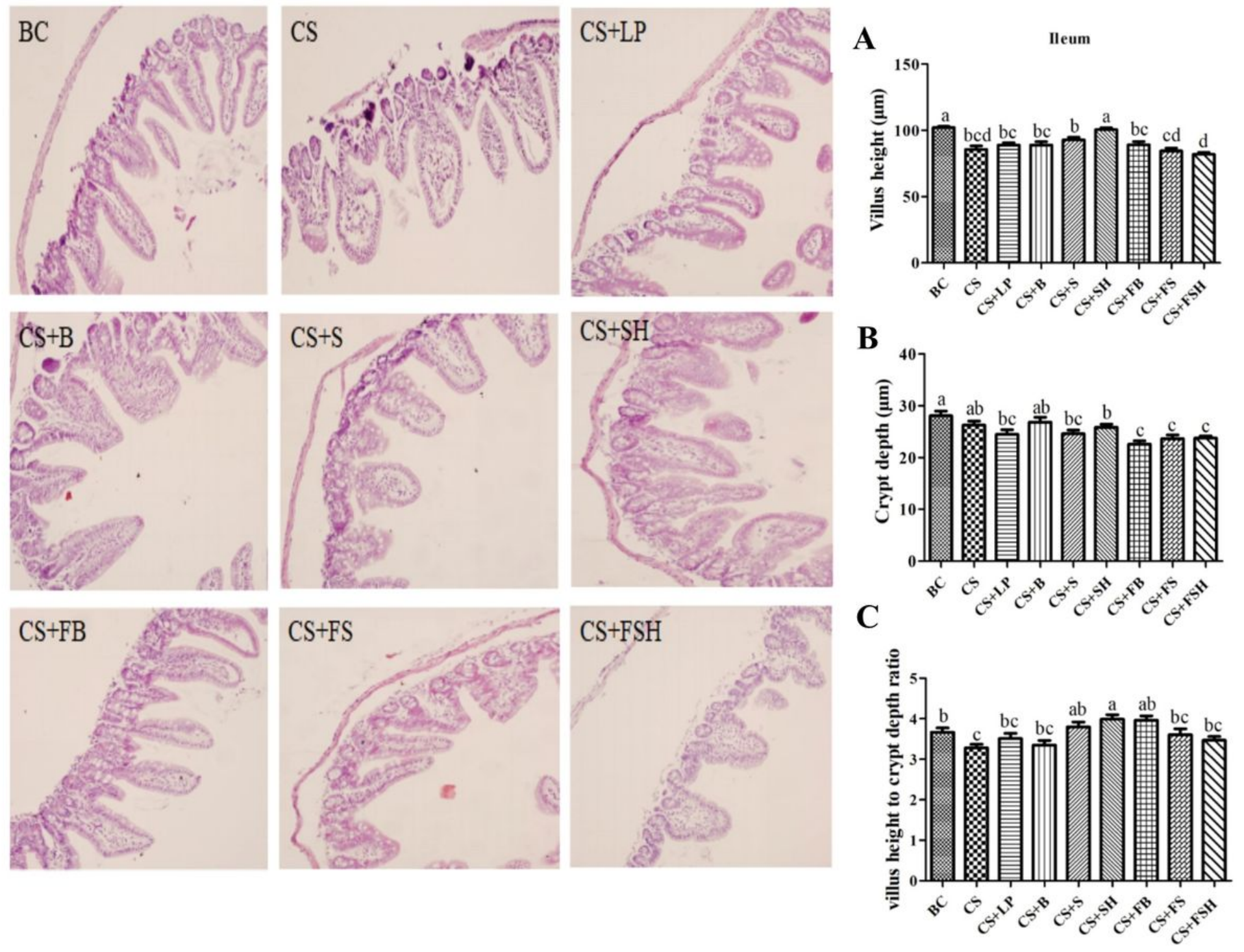

\section{Figure 5}

Intestinal morphology (200x) of ileum in mice with dysbacteriosis induced by ceftriaxone sodium and the alleviation of treatments. A: villus height, B: crypt depth, C: villus height to crypt depth ratio (VH/CD). BC: Blank Control, CS: Ceftriaxone Sodium, CS+LP: Ceftriaxone Sodium + Lactobacillus Plantarum, CS+B: Ceftriaxone Sodium + Buzhongyiqi decoction, CS+S: Ceftriaxone Sodium + Sijunzi decoction, CS+SH: Ceftriaxone Sodium + Shenlingbaizhu decoction, CS+FB: Ceftriaxone Sodium + Fermented Buzhongyiqi decoction, CS+FS: Ceftriaxone Sodium + Fermented Sijunzi decoction and CS+FSH: Ceftriaxone Sodium + Fermented Shenlingbaizhu decoction. $n=3$. Mean values with unlike letters were significantly different $(P<0.05)$. 

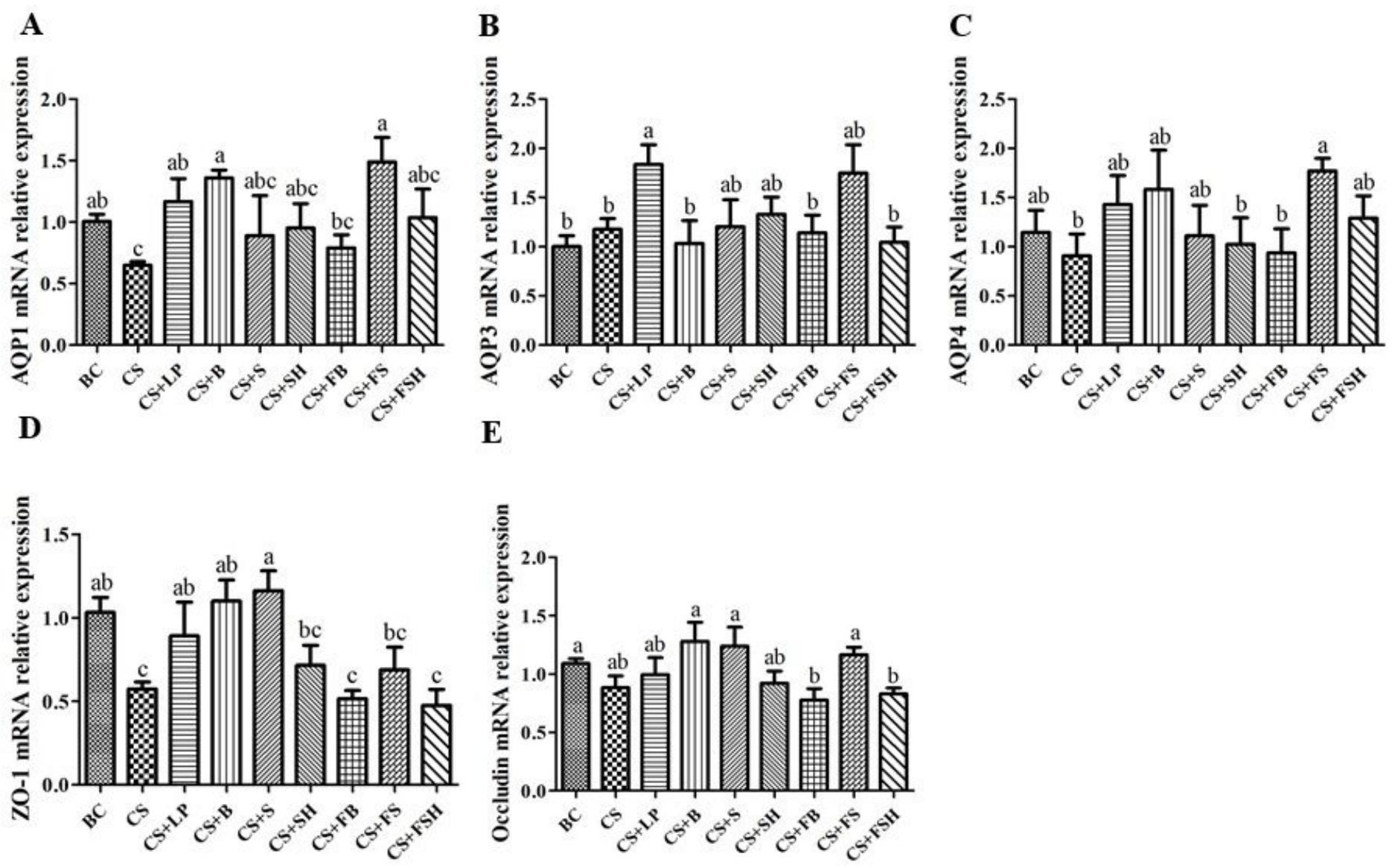

Figure 6

The mRNA relative expression levels of AQP1, AQP3, AQP4, ZO-1, and Occludin genes of duodenum in mice with dysbacteriosis induced by ceftriaxone sodium and the alleviation of treatments (A-E). AQP, Aquaporins; ZO-1, Zonula occludens-1. BC: Blank Control, CS: Ceftriaxone Sodium, CS+LP: Ceftriaxone Sodium + Lactobacillus Plantarum, CS+B: Ceftriaxone Sodium + Buzhongyiqi decoction, CS+S:

Ceftriaxone Sodium + Sijunzi decoction, CS+SH: Ceftriaxone Sodium + Shenlingbaizhu decoction, CS+FB: Ceftriaxone Sodium + Fermented Buzhongyiqi decoction, CS+FS: Ceftriaxone Sodium + Fermented Sijunzi decoction and CS+FSH: Ceftriaxone Sodium + Fermented Shenlingbaizhu decoction. $n=5$. Mean values with unlike letters were significantly different $(P<0.05)$. 

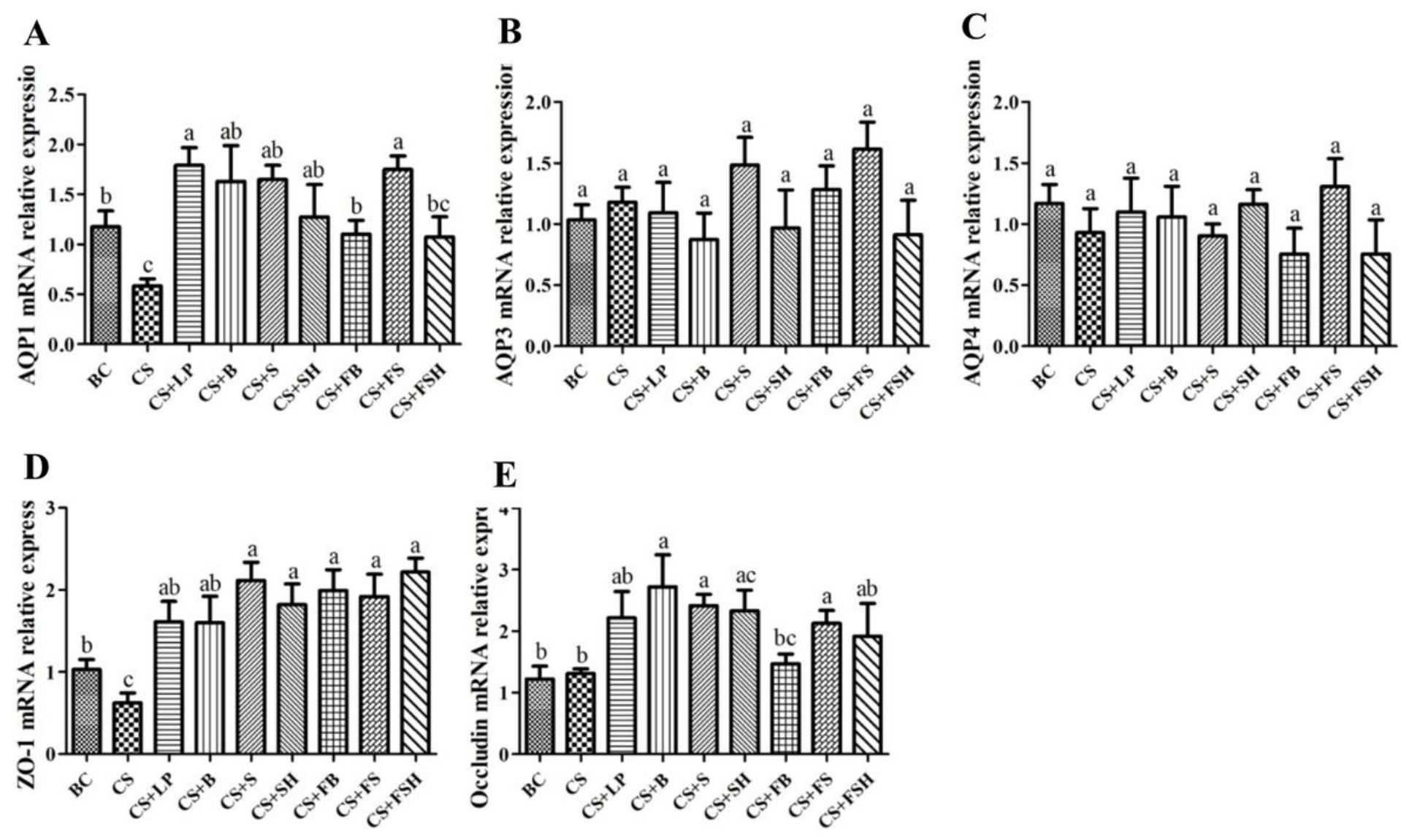

Figure 7

The mRNA relative expression levels of AQP1, AQP3, AQP4, ZO-1, and Occludin genes of colon in mice with dysbacteriosis induced by ceftriaxone sodium and the alleviation of treatments (A-E). AQP, Aquaporins; ZO-1, Zonula occludens-1. BC: Blank Control, CS: Ceftriaxone Sodium, CS+LP: Ceftriaxone Sodium + Lactobacillus Plantarum, CS+B: Ceftriaxone Sodium + Buzhongyiqi decoction, CS+S:

Ceftriaxone Sodium + Sijunzi decoction, CS+SH: Ceftriaxone Sodium + Shenlingbaizhu decoction, CS+FB: Ceftriaxone Sodium + Fermented Buzhongyiqi decoction, CS+FS: Ceftriaxone Sodium + Fermented Sijunzi decoction and CS+FSH: Ceftriaxone Sodium + Fermented Shenlingbaizhu decoction. $n=5$. Mean values with unlike letters were significantly different $(P<0.05)$. 
A

PCA

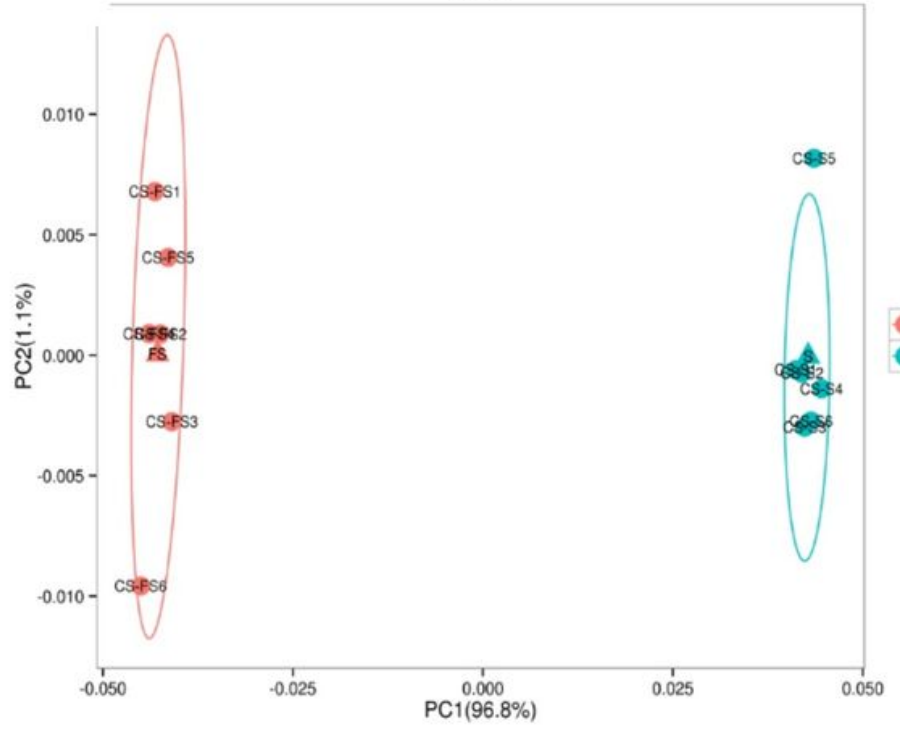

B

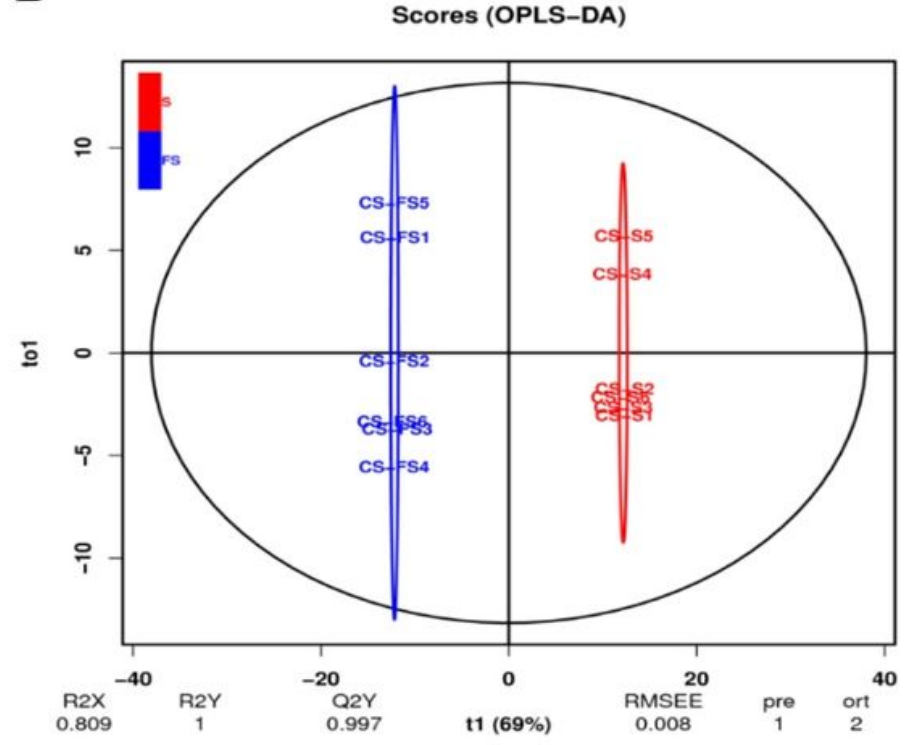

\section{Figure 8}

The score plots of Principal Component Analysis (PCA) (A) and Orthogonal Partial Least SquaresDiscriminant Analysis (OPLS-DA) (B) of Sijunzi tang (S) and Fermented Sijunzi tang (FS).
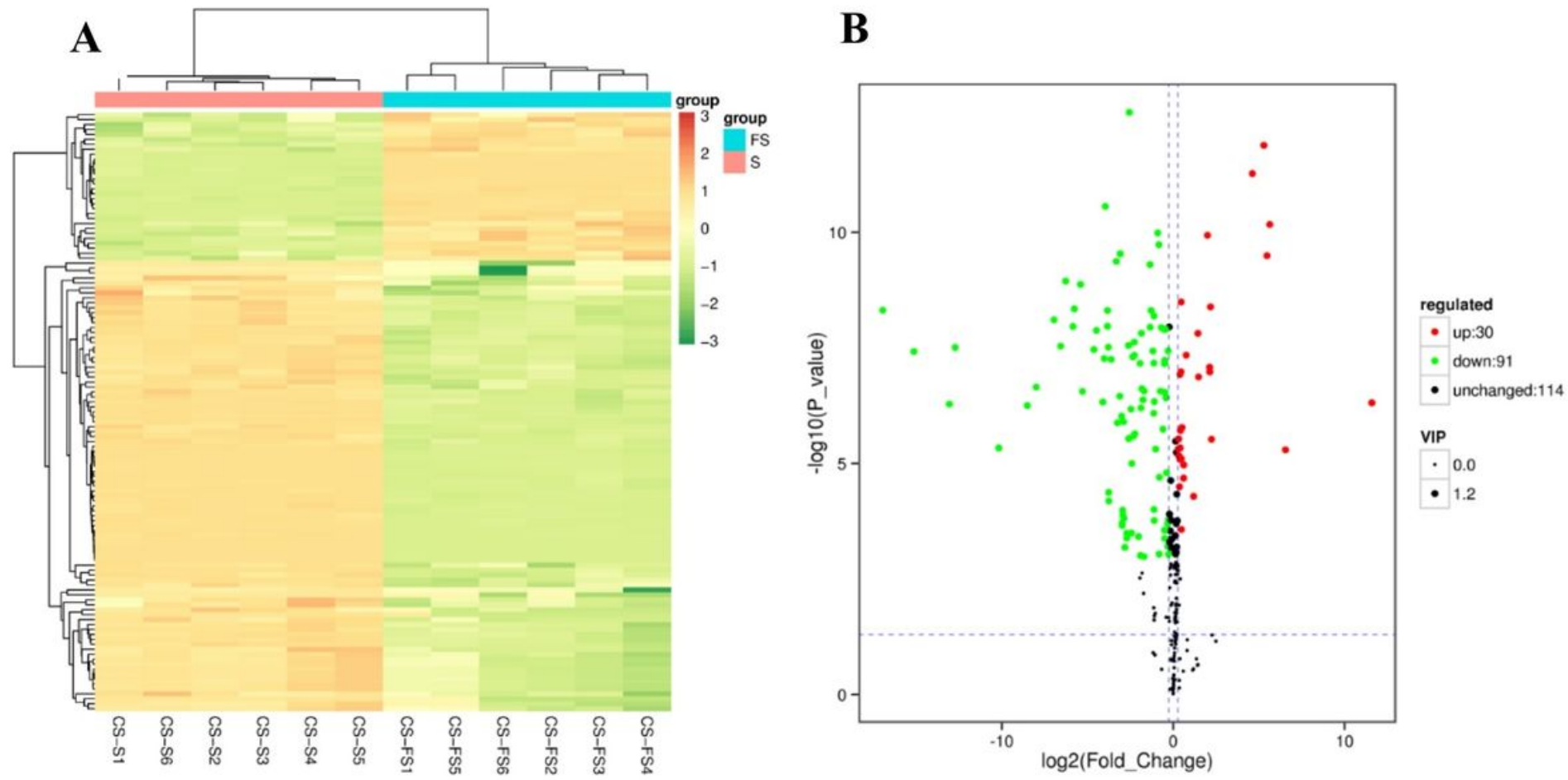

Figure 9

The heat maps (A) and volcano plots (B) of significantly different compounds in Sijunzi tang (S) and Fermented Sijunzi tang (FS). The red spots indicate significantly up-regulated compounds, and the green spots represent significantly down-regulated compounds. 


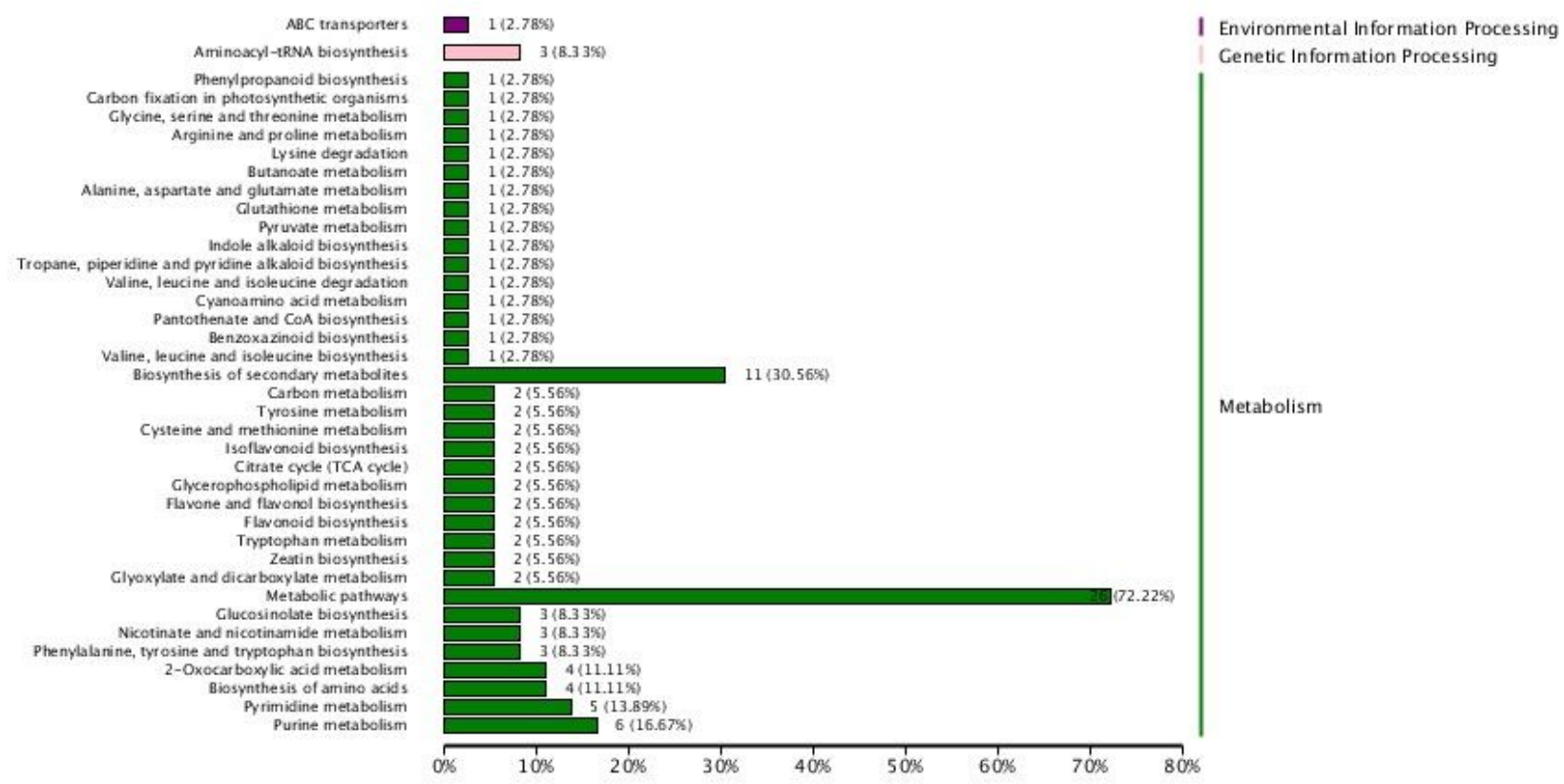

Figure 10

Kyoto Encyclopedia of Genes and Genomes (KEGG) pathway classification maps of significantly different compounds between Sijunzi tang (S) and Fermented Sijunzi tang (FS). The number behind the pathway represents the amount of significantly different compounds in this pathway; the percentage indicates the ratio of amount of significantly different compounds in the pathway to all significantly different compounds.

\section{Supplementary Files}

This is a list of supplementary files associated with this preprint. Click to download.

- supplementaryfile1.xls

- supplementaryfile2.xls 